\title{
Politiques de L'enseignement Supérieur au
} Brésil et en Suisse

\author{
Políticas de Educação Superior no Brasil e na Suíça \\ Politicas para la Educación Superior en Brasil y en Suiza
}

MARIA LOURDES GISI ${ }^{a}$

ISABEL VOIROL-RUBIDO ${ }^{b}$

\section{Résumé}

Cet article examine les politiques de l'enseignement supérieur au Brésil et en Suisse, dans le but d'identifier de possibles similitudes dans la mise en place de ces politiques. L'étude a été réalisée au travers de l'analyse de documents portant sur les politiques de l'enseignement supérieur des deux pays. Avec les réformes effectuées à partir des années 1990, les deux pays ont suivi des chemins différents, la Suisse ayant adhéré au processus de Bologne. Ce fait indique déjà des différences accentuées, mais on peut constater quelques similitudes, que nous avons également rencontrées dans des documents en provenance d'organismes internationaux et de conférences sur l'enseignement supérieur, qui mettent en évidence l'impact de la globalisation sur les différents secteurs de la société. On remarque des similitudes dans l'organisation de l'enseignement supérieur, en particulier en ce qui concerne la diversification institutionnelle et les exigences d'évaluation institutionnelle. Néanmoins, en ce qui concerne la mobilité étudiante, on constate une différence significative, le Brésil ne comptant encore que peu d'étudiants étrangers par rapport à l'ensemble des étudiants immatriculés. Différence marquée, également, en ce qui concerne le financement de l'enseignement supérieur, considérant qu'au Brésil le système est largement privé,

\footnotetext{
a Pontifícia Universidade Católica do Paraná, Curitiba, PR, Brasil. Doutorado em Educação Brasileira, e-mail: maria.gisi@pucpr.br

b Université de Genève, Genève, Suisse. Doctorat en Sciences de l'éducation, e-mail: maria-isabel.voirol-rubido@unige.ch
} 
au contraire de la Suisse où la part du financement privé dans l'enseignement supérieur n'est que de $20 \%$. Les réformes effectuées sont nombreuses, mais demeure la question de la finalité de l'enseignement supérieur qui, même dans sa dimension professionnelle ne saurait se limiter aux exigences du monde du travail, mais avoir pour finalité une éducation émancipatrice.

Mots-clés: Politiques Éducationnelles. Enseignement Supérieur. Réforme de L'enseignement.

\section{Resumo}

O objeto deste artigo são as políticas de educação superior do Brasil e da Suíça e tem como objetivo identificar semelhanças decorrentes da implementação dessas políticas. O estudo foi realizado mediante análise de documentos relativos às políticas para a educação superior existentes nos dois países. Com reformas efetuadas a partir da década de 1990, os dois países trilharam caminhos diferentes quando a Suíça aderiu ao Processo de Bolonha. Tal fato já indica diferenças acentuadas, mas pode-se constatar algumas semelhanças que encontram-se também em documentos oriundos de organismos internacionais e conferências sobre a educação superior, evidenciando o impacto da globalização nos diferentes setores sociais. Observa-se que existem semelhanças na organização da educação superior, em especial no que se refere à diversificação institucional e nas exigências de avaliação institucional. No entanto, no que se refere à mobilidade estudantil, se constata uma diferença significativa, pois o Brasil ainda se encontra com poucos estudantes estrangeiros em relação ao total de estudantes matriculados. Há também uma diferença acentuada no que se refere ao financiamento da educação superior, considerando que no Brasil o sistema é majoritariamente privado, ao contrário da Suiça, onde representa apenas $20 \%$ do financiamento da educação superior no país. São muitas as reformas efetuadas, mas permanece o questionamento sobre a finalidade da educação superior, que mesmo na sua dimensão profissional, não pode ficar restrita às exigências do mundo do trabalho, mas ter como finalidade uma educação emancipatória.

Palavras-chave: Políticas Educacionais. Educação Superior. Reforma Educacional.

\section{Resumen}

El objeto de este artículo son las políticas de educación superior de Brasil y Suiza y tiene como objetivo identificar similitudes resultantes de la implementación de estas políticas. El estudio se llevó a cabo mediante el análisis de documentos relacionados con las políticas de educación superior existentes en ambos países. Con reformas llevadas a cabo desde la década de 1990, los dos países siguieron caminos diferentes cuando Suiza se unió al Proceso de Bolonia. Este hecho ya indica marcadas diferencias, pero podemos ver algunas similitudes que también encontramos en documentos de organismos internacionales y conferencias sobre educación superior, destacando el 
impacto de la globalización en diferentes sectores sociales. Se observa que existen similitudes en la organización de la educación superior, especialmente en lo que respecta a la diversificación institucional y los requisitos de evaluación institucional. Sin embargo, con respecto a la movilidad de estudiantes, hay una diferencia significativa, ya que Brasil todavía tiene pocos estudiantes extranjeros en relación al número total de estudiantes matriculados. También hay una marcada diferencia con respecto al financiamiento de la educación superior, considerando que en Brasil el sistema es mayoritariamente privado, al contrario de Suiza, donde representa solo el $20 \%$ del financiamiento de la educación superior en el país. Son muchas las reformas llevadas a cabo, pero queda la pregunta sobre el propósito de la educación superior, que, incluso en su dimensión profesional, no puede restringirse a las exigencias del mundo laboral, sino tener como finalidad una educación emancipadora.

Palabras clave: Políticas Educativas. Educación Universitaria. Reforma Educativa.

\section{Introduction}

Cet article traite des politiques de l'enseignement supérieur au Brésil et en Suisse, examinées selon différents points de vue, ainsi que des implications de ces politiques pour le procès de formation. Il examine aussi l'organisation institutionnelle et les systèmes d'évaluation institutionnelle, dans le but de mettre à jour d'éventuelles similitudes entre les deux pays.

L'étude a été réalisée à partir de l'analyse de documents relatifs aux politiques de l'enseignement supérieur des deux pays. Il ne s'agit pas d'effectuer une analyse comparative, mais seulement de présenter deux réalités sous l'angle de leurs politiques éducationnelles, issues de pays fortement contrastés en termes de population et de caractéristiques économiques et socioculturelles. Cependant, considérant l'impact de la globalisation, qui se fait sentir dans tellement de domaines, et pas seulement dans le domaine économique, cette recherche nous a paru pertinente.

\section{La Politique Suisse de Formation Supérieure}

La Suisse, petit pays d'Europe Occidentale composé de vingt-six cantons, doté d'une démocratie directe et d'une organisation politique décentralisée, s'est 
engagée depuis une trentaine d'années dans une réforme de son système de formation supérieure pour répondre aux exigences d'une société de plus en plus complexe (SUISSE, 2002). Son système de formation supérieure englobe les hautes écoles universitaires, les hautes écoles spécialisées, les hautes écoles pédagogiques ainsi qu'une offre de formation professionnelle supérieure destinée aux personnes qualifiées désireuses d'élargir leurs qualifications.

Cette partie présente brièvement les grandes lignes actuelles de la politique suisse de formation supérieure.

La politique suisse de formation supérieure se caractérise de nos jours par:

- une volonté de définir une stratégie de formation supérieure commune nationale tout en préservant l'autonomie des cantons pour garantir une gouvernance de sa qualité,

- un financement de plus en plus orienté vers l'encouragement à fournir certaines prestations en fonction des besoins avec la volonté de développer un lien étroit entre la recherche et la société en valorisant économiquement les savoirs et en gérant la propriété intellectuelle, et

- une internationalisation marquée pour rester dans la course de la compétition internationale,

- une forte diversification de l'offre de formation pour répondre à la demande croissante de la population que ce soit en termes de contenus de formation ou de volume,

Dès les années 2000, l'offre de formation tertiaire en Suisse s'est diversifiée afin de répondre au mieux aux exigences d'une société de plus en plus complexe et d'une économie internationalisée extrêmement concurrentielle avide d'innovation et de compétences de plus en plus pointues ${ }^{1}$. Elle se concrétise aujourd'hui dans

\footnotetext{
${ }^{1}$ La Suisse investit 3\% de son PIB dans la recherche et le développement la plaçant au sixième rend des pays qui investissent le plus dans ce domaine. Les principaux domaines d'investissement sont l'industrie pharmaceutique et chimique (34\%), l'industrie mécanique et métallurgique $(16 \%)$, les laboratoires de recherche $(14 \%)$ et les nouvelles technologies (11\%). Source : Office fédérale de la statistique, site internet https://www.eda.admin.ch/aboutswitzerland/fr/home/wirtschaft/uebersicht/forschungund-entwicklung.html. Consulté le 26 août 2020.
} 
plusieurs types d'institutions à vocations différentes. Les hautes écoles universitaires regroupant les universités et les écoles polytechniques fédérales s’investissent dans la recherche fondamentale et l'enseignement de disciplines générales (médecine, droit, économie, ingénierie, etc.). Les hautes écoles spécialisées à vocation plus pratique s'investissent dans la recherche appliquée et des professions spécialisées (santé, tourisme, gestion, etc.). Les hautes écoles pédagogiques, qui forment les enseignants, et les écoles professionnelles supérieures, qui s'adressent aux personnes qualifiées souhaitant élargir leurs qualifications, viennent compléter l'offre de formation tertiaire (niveau CITE $5^{2}$ ) tout comme la possibilité offerte par les hautes écoles universitaires d'obtenir un doctorat (niveau CITE 6).

D’un point de vue politique, l'éducation relève d'une prérogative cantonale. En matière d'enseignement supérieur, cependant, Confédération et cantons associent leurs forces pour définir ensemble les orientations stratégiques de la politique en la matière (art. 63a Constitution fédérale de la Confédération suisse). Afin de lui donner les moyens de déployer tout son potentiel, l'enseignement supérieur jouit en Suisse d'une grande autonomie académique, organisationnelle et financière qui varie selon le profil de l'institution (GARCON, 2014).

Le pilotage stratégique des deux écoles polytechniques fédérales, actives dans des domaines d'importance stratégique pour le pays (sciences de la vie, nanotechnologies et sciences de l'information et de la communication) relèvent de la responsabilité de la Confédération alors que les hautes écoles universitaires et les hautes écoles spécialisées sont pilotées par les cantons. La formation professionnelle supérieure relève pour sa part d'un partenariat entre la Confédération, les cantons et les organisations du monde du travail (SUISSE, 2019).

Cette forme d'organisation de l'enseignement supérieur est le fruit d'un important processus de réforme entrepris à partir des années 1990 visant à développer une offre de formation capable de répondre aux exigences du monde actuel en attirant les meilleurs enseignants et chercheurs dans un contexte d'internationalisation. Ce processus, toujours en cours de nos jours, vise à davantage d'autonomie pour les hautes écoles tout en centralisant les lignes directrices, une

\footnotetext{
${ }^{2}$ Classification internationale type de l'éducation.
} 
harmonisation de la structure des formations supérieures, une mise en réseau des acteurs de l'enseignement supérieur, l'octroi de subventions en fonction de la qualité des prestations fournies, un renforcement de la collaboration entre la Confédération et les cantons en matière d'enseignement supérieur ainsi que par un soutien financier de la Confédération à des projets jugés d'importance nationale tels que, par exemple, la mise en oeuvre d'un campus virtuel mobilisant les technologies de l'information et de la communication ou un programme d'égalité des chances entre les sexes visant à promouvoir les carrières académiques féminines.

La recherche accrue d'autonomie se concrétise par la dotation d'un mandat de prestations à l'institution de formation et d'un budget global que la haute école gère selon sa propre stratégie. Cette marge de manoeuvre offerte aux hautes écoles a impliqué que soit mis en place dès 2001 un Organe indépendant d'accréditation et d'assurance qualité (OAQ) ayant pour mission d'assurer la qualité des activités menées dans l'enseignement supérieur et d'accréditer les filières de formation et les hautes écoles.

Les deux écoles polytechniques fédérales sont majoritairement financées par la Confédération. Les autres le sont en grande partie directement par les cantons et indirectement par la Confédération sous forme de subventions (env. 25\%). A cela s'ajoutent les fonds fédéraux dédiés à la recherche gérés par le Fonds national suisse de recherche scientifique (FNS) ou la Commission pour la technologie et l'innovation (CTI), bailleurs de fonds des différents projets de recherche mis en oeuvre par les institutions requérantes. Il convient également de préciser que bien que les taxes universitaires à la charge des étudiants aient eu tendance à augmenter ces dernières années, la part du financement privé de l'enseignement supérieur suisse (taxes universitaires et investissements privés des entreprises) reste marginale (SUISSE, 2006 p. 35 ; SUISSE, 2018, p. 209;). Il ne représente que 20\% de l'ensemble du financement (SUISSE, 2018, p. 182).

La Suisse a par ailleurs adhéré à la Déclaration de Bologne qui prévoit l'harmonisation à l'échelle européenne non seulement de la structure des études tertiaires en trois paliers (bachelor, master et doctorat) mais également les contenus et les formes d'enseignement afin de faciliter la reconnaissance des diplômes et la mobilité des chercheurs, des enseignants et des étudiants. Elle a également mis en 
place une procédure d'assurance qualité et d'accréditation calquée sur les critères et les standards européens (Loi fédérale sur l'encouragement et la coordination des hautes écoles, LEHE).

La mise en réseau du savoir à l'échelle européenne et plus largement internationale des acteurs des hautes écoles est un enjeu majeur pour un petit pays comme la Suisse, qui plus est à la pointe de l'innovation dans de nombreux domaines $^{3}$. Elle participe ainsi à l'espace européen de l'enseignement supérieur et de la recherche en adhérant à de nombreux programmes de recherche et de développement (p. Ex. Socrates, Léonard de Vinci, Erasmus), elle renforce sa coopération avec les pays voisins en signant avec eux des Accrods bilatéraux qui visent notamment la reconnaissance des diplômes, et soutient la coopération scientifique et technologique internationale de l'enseignement supérieur. Elle s'inscrit ainsi de plain-pied dans le mouvement d'internationalisation de l'enseignement supérieur.

Au vu du bref panorama qui précède, il convient d'une part de relever une internationalisation croissante du système de formation supérieure suisse "[qui] recouvre [...] à la fois les processus de globalisation et d'européanisation, inscrits dans leurs dynamiques autant cognitives qu'économiques, politiques, sociales ou culturelles" (LERESCHE et al., 2009, p. 4) et de souligner l'imprégnation libérale (GOHIER, 2016) d'une politique suisse visant l'excellence de son enseignement supérieur dans le but d'augmenter son potentiel d'innovation scientifique et sa compétitivité visant des performances économiques (BEHRENS \& BOURGOZ FROIDEVAUX, 2016). S’il est avéré que ce processus d'internationalisation contribue à l'amélioration de la qualité du système d'enseignement supérieur en Suisse (SER, 2006), ne risque-t-on pas également à travers la réforme en cours de l'enseignement supérieur d'induire des inégalités (entre acteurs, chercheurs, institutions) et des pressions susceptibles d'entacher l'efficacité du système ? La question est posée. Rappelons que plus que jamais "réformer l'éducation [implique]

\footnotetext{
3 https://www.eda.admin.ch/aboutswitzerland/fr/home/wirtschaft/uebersicht/forschungund-entwicklung.html, site internet consulté le 27 août 2020.
} 
de travailler ensemble au bien commun en développant une intelligence collective" (DE KETELE, 2020).

\section{La Politique d'Évaluation de L'enseignement Supérieur Suisse}

L'intégration de la Suisse à l'Espace européen de l'enseignement supérieur en signant notamment la Déclaration de Bologne l'engage à mettre en place et à respecter des procédures d'assurance qualité et d'accréditation de ses institutions d'enseignement supérieur (SUISSE, 2002). Ainsi, la loi sur l'encouragement et la coordination des hautes écoles (LEHE), entrée en vigueur en 2015, impose désormais à toutes les institutions de l'enseignement supérieur, qu'elles soient publiques ou privées, qu'elles dépendent de l'autorité fédérale ou cantonale, l'obligation de se soumettre à une accréditation institutionnelle afin d'obtenir le droit d'appellation d"'université", de "haute école spécialisée" ou de "haute école pédagogique" et de recevoir les contributions fédérales.

L'accréditation institutionnelle vise à garantir la qualité de l'enseignement, de la recherche et des prestations de services des institutions de l'enseignement supérieur. Ces dernières sont responsables de l'assurance et du développement de la qualité de leurs prestations et ont le devoir de transparence et de rendre des comptes (accountability) dans la lignée du courant de la Nouvelle gestion publique importée du secteur privé (GRUENING, 2001; HOOD, 1991).

La décision d'accréditation est prise par le Conseil suisse d'accréditation au terme d'un processus qui examine dans le détail le fonctionnement de l'institution candidate ayant formulé la demande. Les standards de qualité retenus par le Conseil suisse d'accréditation concernent la stratégie d'assurance de la qualité, la gouvernance, l'enseignement, la recherche et les prestations de services, les ressources ainsi que la communication interne et externe.

Le processus d'accréditation comprend quatre étapes majeures mobilisant les acteurs internes et externes à l'institution requérante ainsi que l'Agence d'accréditation mandatée par le Conseil suisse d'accréditation pour mener à bien la procédure (SUISSE, 2015). Après avoir soumis une demande d'accréditation au 
Conseil suisse d'accréditation qui la valide, l'institution requérante entre dans l'étape 1 de la procédure consistant à se soumettre à une évaluation interne (autoévaluation) de la qualité de ses prestations. Cette étape consiste à rendre compte de la qualité de ses prestations en s'appuyant sur des données récoltées auprès de tous les groupes représentatifs de la haute école, à savoir les personnels d'enseignement, de recherche, administratif et technique ainsi que les étudiants. Il s'agit d'un processus participatif transparent auquel tous les acteurs de l'institution sont conviés au terme duquel un rapport d'autoévaluation est produit sur la base de données telles que les évaluations des cours, des enseignants, des étudiants et de données issues de statistiques dans le but d'évaluer le degré de conformité aux standards de qualité recherchés. Ce rapport présente les caractéristiques de l'institution, décrit le processus d'autoévaluation et le système d'assurance qualité et évalue la réalisation des standards de qualité recherchés. Il présente les points forts, les points faibles, les défis et les possibilités de développement possible ainsi qu'un plan d'action pour le développement des systèmes d'assurance qualité. Un regard réflexif et critique est attendu de la part de l'insititution soumise à autoévaluation.

L'Agence d'accréditation mandate ensuite une évaluation externe des standards d'assurance qualité par au moins cinq experts dotés des compétences, de l'expérience et de l'impartialité nécessaires pour assumer cette deuxième étape. Les experts se basent sur le rapport d'autoévaluation produit par l'insitution requérante et leurs propres observations. Ces dernières résultent des visites qu'ils rendent à l'institution d'enseignement durant lesquelles les experts organisent des entretiens avec les différentes instances de l'institution.

En se basant sur le rapport d'autoévaluation et le rapport des experts externes, l'Agence d'accréditation soumet au Conseil suisse d'accréditation une proposition d'accréditation. Ce rapport est également soumis à l'insitution requérante pour une prise de position. A la suite de quoi l'Agence d'acréditation peut, si elle le souhaite, modifier sa proposition en tenant compte des arguments émis par la haute école.

Finalement, le Conseil suisse d'accréditation prend sa décision en se basant sur le rapport d'autoévaluation, sur le rapport des experts externes, de la décision de l'Agence d'accréditation et de la position de l'insitution requérante. L'accrédiation 
peut alors être accordée sans conditions, avec conditions ou refusées. Un droit de recours est prévu à l'art. 65 al. 2 LEHE. Lorsque l'accréditation est acceptée sous conditions, l'institution requérante dispose d'un délai pour la mise en conformité. L’Agence d'accrédiation est alors mandatée pour contrôler cette mise en conformité.

La durée de l'accréditation est de sept ans au terme desquels une procédure de renouvellement est engagée. Les insitutions accréditées figurent sur une liste publiée par le Conseil suisse d'accréditation.

\section{Politiques éducationnelles de l'enseignement supérieur au Brésil}

L'enseignement supérieur au Brésil a acquis de nouvelles caractéristiques après l'approbation de la loi des directives et bases de l'Éducation nationale - Loi no 9394/96, qui a organisé l'éducation du pays en deux niveaux : l'éducation de base et l'enseignement supérieur.

C'est avec cette loi et sa législation complémentaire que s'explicite pour l'enseignement supérieur la diversification des formations et des institutions, la définition de normes de qualité de l'enseignement et l'accent mis sur l'évaluation institutionnelle. Conformément à l'article 45, «l'enseignement supérieur sera dispensé dans des institutions d'enseignement supérieur, publiques ou privées, avec des degrés variables de spécialisation »(BRASIL, 1996).

Outre les institutions publiques (fédérales, d'États et municipales) et privées à but lucratif ou non, le pays présente une diversification des types d'institution, qui se divisent entre : universités, centres universitaires, instituts fédéraux et facultés. Il y avait, en 2018, selon le Recensement de l'enseignement supérieur, 2.537 institutions, dont $88,2 \%$ étaient privées et $11,8 \%$ publiques $; 75,4 \%$ des 8.450 .755 immatriculations concernaient des institutions privées. Les universités totalisaient $52,9 \%$ de toutes les immatriculations, ce qui est important puisque celles-ci possèdent une structure et sont soumises à des exigences légales qui favorisent la qualité de l'enseignement. Quant aux autres, 22,6\% étaient des centres universitaires, $22,2 \%$ des facultés et seulement 2,3\% des Instituts fédéraux et Cefets (Centres fédéraux d'enseignement technique). Pour ce qui est des types de formation, 58\% 
sont des licences, 20,9\% des formations techniques et 20,5\% des licences pour l'enseignement. De nombreux étudiants travaillent de jour et étudient le soir, un total de 42,4\%, alors que 58,6\% fréquentent les cours diurnes (BRASIL, 2019).

L'enseignement supérieur étant en majorité privé, des programmes ont été créés pour en faciliter l'accès et y garantir la permanence. L'un de ces programmes est le Programme université pour tous - PROUNI, institué par la Loi no 11.096/2005, qui concerne les institutions privées, qui sont exemptées de certains impôts en échange de bourses pour les étudiants. Il existe également un financement de l'enseignement par le FIES - Fond de financement étudiant, créé par la Loi n ${ }^{\circ}$ 10.260/2001, qui octroie des bourses aux étudiants immatriculés dans les institutions privées d'enseignement supérieur ayant adhéré au programme et qui bénéficient d'une évaluation positive lors du processus d'évaluation institutionnelle conduit par le Ministère de l'éducation - MEC (BRASIL, 2001, 2005).

Toujours en ce qui concerne l'élargissement de l'accès, l’Université ouverte du Brésil - UAB - décret $n^{\circ} 5.800 / 2006$, propose des formations à distance. Le Programme de soutien aux plans de restructuration et d'expansion des universités fédérales - REUNI, créé par le décret no 6.096/2007, est aussi approuvé. C’est à partir de ce programme qu'a été élargi l'accès à l'enseignement supérieur, par la création de nouvelles institutions, par l'occupation des places vacantes, par l'amélioration des programmes et de l'infrastructure, par la réduction des taux d'abandon, l'augmentation du nombre d'admissions, en particulier en période nocturne (cours du soir), l'élargissement des politiques d'inclusion et d'aide aux étudiants, l'articulation entre les trois cycles universitaires et celle de l'enseignement supérieur à l'enseignement élémentaire. La loi des quotas - Loi no 12.711/2012 est également créée, qui détermine qu'un minimum de 50\% des places pour les licences et formations techniques dans les institutions fédérales d'enseignement supérieur soient destinées aux étudiants provenant d'écoles publiques (BRASIL, 2006, 2007, 2012).

Selon la Loi no $13.409 / 2016$, art. $3^{e}$ :

[...] Dans chaque institution fédérale d'enseignement supérieur, les places seront attribuées, par programme et par horaire, à des étudiants autodéclarés comme noirs, mulâtres et indigènes et par des personnes handicapées, conformément à la 
législation, en proportion du nombre total de places, au minimum égal à la proportion [...] de l'État où est installée l'institution, selon le dernier recensement de I'IBGE (BRASIL, 2016).

L'effort pour augmenter le nombre de places n'a cependant pas été suffisant pour atteindre les objectifs établis par le Plan national de l'éducation, qui propose un taux net de 33\% des immatriculations dans l'enseignement supérieur des jeunes de 18 à 24 ans; selon le Recensement de l'enseignement supérieur, le pays à atteint le taux de 21,7\% (BRASIL, 2014;2019).

La reformulation de l'enseignement supérieur s'est intensifiée, particulièrement après l'approbation de la Loi des directives et bases de l'Éducation nationale - LDBEN - Loi no 9394/96 et en conséquence de l'approbation de résolutions, décrets, arrêtés, etc. dans le but, selon Catani et Oliveira (2002, p. 12), de créer « [...] un système basé sur la flexibilité, la compétitivité, la différentiation et l'évaluation ».

Les politiques de l'enseignement supérieur au Brésil, plus actives, sous plusieurs aspects, au cours des dernières années, ont suscité des transformations liées, principalement à l'expansion du secteur privé à but lucratif. Dans ce contexte s'est manifestée une préoccupation pour les politiques d'accès et d'évaluation. Ces changements ont pris place dans le contexte de crise générale du capitalisme qui s'est déclaré dans les années 1970 et des transformations qui se sont produites avec la globalisation de l'économie, visant la réorganisation du système capitaliste.

C’est à partir de cette époque que, selon Catani et Oliveira (2002, p. 11-12), commence l'intensification de l'internalisation du capital financier et la mise en place du modèle de l'accumulation flexible du capital, dans le contexte de l'adoption des politiques néolibérales. La privatisation et la marchandisation de l'enseignement supérieur, selon les termes de Sguissardi (2016, p. 22) «[...] ont trouvé un terrain particulièrement fertile dans les années 1990, lorsqu'un national-développementisme précaire est remplacé par le soi-disant néolibéralisme ».

L'enseignement supérieur est devenu alors la cible de changements portant sur sa place dans un monde bouleversé par les avancées de la technologie et de la communication, dans le contexte de l'implantation du projet néolibéral. À partir de là ont surgi les mises en questions de l'efficience, de la qualité, de la compétitivité et 
de l'équité de l'enseignement, ainsi que sur les changements affectant les profils professionnels et, par conséquent, le processus de formation, comme sur le rôle de l'éducation permanente, l'autonomie des sciences et la liberté académique, le rapport entre l'université et le secteur productif.

Dans le paysage de transformation de la société contemporaine, mis sous pression par l'ordre productiviste du monde globalisé, l'enseignement supérieur est encore une fois poussé à participer au processus, dans le sens de se mettre à jour face aux défis d'ordre social ou économique. Une convergence indéniable, qui se produit au niveau mondial, concerne l'axe orientant tous ces changements, qui se situent dans la dimension économiciste, néolibérale, imposée par des organismes internationaux comme la Banque mondiale, qui sont en parfait accord avec la norme de la globalisation (BASTOS, 2009, p. 160).

En ce qui concerne l'évaluation, Dourado (2002, p. 243) mentionne qu'elle était comprise comme l'un des axes structurant des politiques éducatives contemporaines, liées aux changements dans le domaine économique et qu'elle s'opposait à un processus évaluatif émancipateur, qui contribue au développement des institutions d'enseignement supérieur.

\section{Politiques d'évaluation de l'enseignement supérieur au Brésil}

Avec l'approbation de la Loi des directives et bases de l'Éducation nationale - LDBEN - Loi no 9394/96, l'évaluation systémique s'est vue attribuer, selon Sousa et Rocha (2018, p. 161), un rôle central comme mécanisme de régulation des politiques éducationnelles. Inscrite dans son article 46, la LDB explicite l'évaluation comme processus régulier, indiquant que l' «autorisation et la reconnaissance des diplômes, comme l'accréditation des institutions d'enseignement supérieur auront des échéances limitées et périodiquement renouvelables, après un processus régulier d'évaluation ». (BRASIL, 1996).

Lorsque la LDB a été approuvée, durant le mandat de Fernando Henrique Cardoso, on avait déjà institué l'Examen national des programmes, connu comme provão, approuvé par la loi no 9.131/95, en vigueur de 1996 à 2003 et qui, selon Dias Sobrinho (2010, pp. 203 et 205), a été « [...] graduellement érigé comme instrument 
central d'évaluation de l'enseignement supérieur brésilien. [...] au contraire de ce qui était dit, le provão n'évaluait pas l'apprentissage ; il mesurait des performances... » Comme le montre l'auteur, le Décret 2.026/96, sous l'inspiration du modèle proposé par l'OCDE, de caractère économiciste, privilégiait les indicateurs suivants :

[...] taux bruts et nets d'immatriculation, disponibilité de places pour les nouveaux étudiants, taux d'abandon et de réussite, temps moyen d'achèvement des études, niveaux de qualification des enseignants, nombre d'étudiants par professeur, taille moyenne des classes, coût par étudiant, pourcentage des coûts de l'enseignement supérieur au total des dépenses de l'éducation publique, pourcentage du PIB dépensé pour l'enseignement supérieur (DIAS SOBRINHO, 2010, p. 2004).

Les résultats obtenus lors du processus évaluatif, et spécialement au «provão» servaient de base aux actes règlementaires d'accréditation et de réaccréditation des institutions et de reconnaissance des programmes.

En 2003, sous le gouvernement de Luiz Inácio Lula da Silva, des changements dans la politique de l'enseignement supérieur ont été effectués, entre autre la création du Système national d'évaluation de l'enseignement supérieur — SINAES, à partir d'une proposition élaborée par la Commission spéciale d'évaluation, créée par le Secrétariat à l'enseignement supérieur. Elle avait pour objectif l'amélioration de la qualité de l'enseignement supérieur et de son engagement social, dans le but d'articuler la réglementation de l'enseignement supérieur à la dimension d'émancipation des institutions d'enseignement supérieur, à partir d'une conception processuelle de l'évaluation.

Le SINAES a été créé par la Loi no 10.861/04 et son article premier établit comme objectif de «garantir le processus national d'évaluation des institutions d'enseignement supérieur, des programmes de licence et des performances académiques de ses étudiants »(BRASIL, 2004). Le Système prévoit une évaluation externe et une évaluation interne (autoévaluation) qui est coordonné par la Commission propre d'évaluation (CPA), favorisant une évaluation plus participative. L'évaluation externe comprend l'Examen national des étudiants (ENADE) et des évaluations des institutions au moyen de visites in loco.

Mais peu à peu les évaluations se sont basées sur les indicateurs résultant de l'ENADE, la note préliminaire du programme (CPC), la note du programme (CC), 
l'indice général du programme (IGC), la note institutionnelle (CI). La proposition initiale de SINAES s'est ainsi perdue au cours des années et l'évaluation a servi toujours davantage au classement des institutions. On observe, dans le contexte actuel, une importance croissante attribuée à l'évaluation de résultats, associée à ce que Ball (2005) appelle le nouveau gestionnariat, qui exprime une politique des néolibéraux et néoconservateurs. Cette sorte d'évaluation exerce une influence sur la gestion des institutions d'enseignement supérieur et sur le procès de formation des jeunes, en influençant directement les lignes directrices des programmes, et donc l'apprentissage des étudiants, ignorant l'autonomie institutionnelle et le point de vue intégral sur le procès d'apprentissage.

Le 15 décembre 2017 a été approuvé le décret n 9.235 qui « détermine l'exercice des fonctions de régulation, de surveillance et d'évaluation des institutions d'enseignement supérieur et des programmes des trois cycle du système fédéral d'enseignement ». Ces décrets s'efforcent d'indiquer toutes les étapes que doit suivre le processus d'évaluation et de régulation, décrivant le processus d'autorisation, d'accréditation et de désaccréditation des institutions d'enseignement supérieur (BRASIL, 2017).

\section{Quelques considérations sur l'enseignement supérieur dans les deux pays}

Les deux pays ont réalisé leurs réformes dans les années 1990 et présentent certaines similitudes que l'on trouve aussi dans les documents provenant d'organismes internationaux et de conférences sur l'enseignement supérieur.

La diversification de l'offre de formations et d'institutions est présente dans les deux pays, bien qu'avec des caractéristiques spécifiques, résultant des directives du Processus de Bologne implanté en Suisse. Il existe dans ce pays des institutions polytechniques fédérales, entretenues par la Confédération, alors que les universités, les écoles de formation des enseignants et les écoles professionnelles sont entretenues par les Cantons, bien que subventionnées à environ 25\% par la Confédération; la part de l'enseignement privé et le paiement de taxes par les étudiants ne représentent que $20 \%$ du financement de l'enseignement supérieur. Au 
Brésil, c'est également à partir des années 1990 que se produit une plus grande diversification des institutions, en universités, centres universitaires, facultés et instituts. En ce qui concerne le financement, il y a, d'un côté, des institutions publiques totalement financée par l'État et, de l'autre, des institutions privées, à but lucratif ou non, avec paiement de mensualités, mais bénéficiant d'exemption d'impôts en échange de l'octroi d'un certain nombre de bourses pour les étudiants, bien qu'en quantité insuffisante. Les données montrent que durant la période de 2005 à 2018, le nombre total de bourses a été de 2.474.435, dont 31\% étaient partielles et 60\% intégrales (BRASIL, 2019b).

L'internationalisation est un objectif pour les deux pays, mais on observe une grande différence en ce qui concerne la mobilité étudiante. Alors que le processus de Bologne a prévu, dans son organisation curriculaire, la possibilité de mobilité étudiante, favorisant la présence d'un nombre considérable d'étudiants d'autres pays dans les institutions d'enseignement supérieur de Suisse, au Brésil, la mobilité étudiante se trouve en phase initiale. Selon le Recensement de l'enseignement supérieur de 2018, il n’y avait que 17.061 étudiants étrangers dans les programmes de licence, provenant de 176 pays, ce qui ne représente que 0,2\% des immatriculations du pays (BRASIL, 2019a).

Bastos (2009, pp.160-62), analysant l'organisation de l'enseignement supérieur au Brésil par rapport aux pays européens, considère que nous ne pouvons pas la comparer à celle des pays européens, qui ont adhéré au processus de Bologne, parce que le pays est davantage aligné sur les documents de l'UNESCO, en particulier la Déclaration mondiale pour l'enseignement supérieur et Paris +5 . Une organisation où s'exprime le plus grand souci de l'exclusion historique des jeunes de l'enseignement supérieur, et donc l'effort d'élargir l'accès et les droits de l'homme. On ne saurait oublier, cependant, que des documents de la Banque mondiale concernant l'enseignement supérieur ont influencé son organisation, en particulier ce qui touche à la diversification institutionnelle. Mais il faut convenir avec l'auteur lorsqu'elle indique, se basant sur Buarque (2003), que nous sommes encore loin d'un système universitaire, du fait, par exemple, des difficultés qui subsistent quant aux transferts et à la mobilité des étudiants, par exemple (BASTOS, 2009, p. 160-62). 
En ce qui concerne l'évaluation institutionnelle, en Suisse, la Loi sur l'encouragement et la coordination des Hautes écoles (LEHE), entrée en vigueur en 2015, oblige toutes les institutions d'enseignement supérieur, qu'elles soient publiques ou privées, à se soumettre à une accréditation institutionnelle pour bénéficier de subventions fédérales.

De même le Brésil dispose d’une législation spécifique pour les actes réglementaires d'accréditation, de réaccréditation d'institutions et de reconnaissance des programmes. Du point de vue de l'implémentation du processus d'évaluation des institutions d'enseignement supérieur, on observe qu'au Brésil certaines époques ont favorisé l'engagement social des institutions envers la société, alors qu'à d'autres moments la préoccupation était centrée sur la mesure de résultats, favorisant ainsi le classement par score.

La question qui demeure est celle des contributions apportées par les réformes du processus de formation professionnelle, comme le rappelle Coelho:

À l'université, on attend de la formation, même dans sa dimension spécialisée et professionnelle, qu'elle ne se perde pas dans les limites étroites d'un entraînement de l'esprit et du corps, en une soi-disant adéquation aux besoins et aux exigences de monde du travail, comme si celles-ci demeuraient statiques (COELHO, 2016, p. 101).

Il est nécessaire que les universités aient toujours pour finalité un processus émancipatoire, dans toutes ses fonctions et ses activités, de façon à donner sens et légitimité à sa praxis (Coelho, 2016).

\section{Référencias}

BALL, S. Profissionalismo, Gerencialismo e Performatividade. Cadernos de Pesquisa, v. 35, n. 126, p. 539-564, 2005.

BASTOS, C. C. B. C. O processo de Bolonha no espaço europeu e a reforma universitária brasileira. In: PEREIRA, E.M.A.; ALMEIDA, M. L. P. Universidade contemporânea: políticas do processo de Bolonha. Campinas: Mercado de Letras, 2009.

BEHRENS, M.; BOURGOZ FROIDEVAUX, A. (eds.) Réflexions sur l'avenir de l'éducation. Quelques jalons et perspectives. Neuchâtel : IRDP éditeur, 2016. 
BRASIL. Lei no 9.394/96, de 20 de dezembro de 1996. Estabelece as diretrizes e bases da educação nacional. Diário Oficial da União, Brasília, DF, 23 de dez. 1996. Disponível em: http://www.planalto.gov.br/ccivil_03/leis/19394.htm. Acesso em: 22 set. 2020.

BRASIL. Ministério da Educação. Lei no 10.260, 12 de julho de 2001. Dispões sobre o Fundo de financiamento ao estudante do Ensino Superior e dá outras providências. Diário Oficial da União, Brasília, DF. Disponível em: http://www.planalto.gov.br/ccivil_03/leis/leis_2001/L10260compilado.htm. Acesso em: 22 set. 2020.

BRASIL. Lei no 10.861, de 14 de abril de 2004. Institui o Sistema Nacional de Avaliação da Educação Superior e dá outras providências. Diário Oficial da União, Brasília, DF, 15 de abr. 2004. Disponível em: http://www.planalto.gov.br/ccivil_03/_ato20042006/2004/lei/110.861.htm. Acesso em: 21 set. 2020.

BRASIL. Lei ${ }^{\circ}$ 11.096, de 13 de janeiro de 2005. Institui o Programa Universidade para Todos - PROUNI, Diário Oficial da União, Brasília, DF, 14 jan. 2005. Disponível em: http://www.planalto.gov.br/ccivil_03/_Ato2004-2006/2005/Lei/L11096.htm. Acesso em: 7 maio 2020 .

BRASIL. Decreto no 5.800, de 8 de junho de 2006. Dispõe sobre o sistema Universidade Aberta do Brasil. Diário Oficial da União, Brasília, DF, 09 de junho de 2005. Disponível em: http://www.planalto.gov.br/ccivil_03/_Ato2004-2006/2006/Decreto/D5800.htm.

Acesso em: 21 set. 2020.

BRASIL. Decreto no 6.096, de 24 de abril de 2007. Institui o Programa de Apoio a Planos de Reestruturação e Expansão das Universidades Federais - REUNI, Diário Oficial da União, Brasília, DF de 25 de abril de 2007. Disponível em: http://www.planalto.gov.br/ccivil_03/_Ato2007-2010/2007/Decreto/D6096.htm.

Acesso em: 21 set. 2020.

BRASIL. Lei 12.711 de 29 de agosto de 2012. Dispõe sobre o ingresso nas universidades federais e nas instituições federais de ensino técnico de nível médio e dá outras providências. Diário Oficial da União, Brasília, DF de 30 de agosto de 2012. Disponível em: http://portal.mec.gov.br/cotas/docs/lei_12711_29_08_2012.pdf. Acesso em: 21 set. 2020.

BRASIL. Lei no 13005 de 25 de junho de 2014. Aprova o Plano Nacional de Educação PNE e dá outras providências. Diário Oficial da União, Brasilia, DF, 26 de jun. 2014. Disponível em: http://pne.mec.gov.br/18-planos-subnacionais-de-educacao/543-planonacional-de-educacao-lei-n-13-005-2014. Acesso em: 21 set. 2020.

BRASIL. Lei $\mathrm{n}^{\circ}$ 13.409, de 28 de dezembro de 2016. Altera a Lei $\mathrm{n}^{\circ} 12.711$, de 29 de agosto de 2012, para dispor sobre a reserva de vagas para pessoas com deficiência nos cursos técnico de nível médio e superior das instituições federais de ensino. Diário Oficial da União, Brasília, DF, 29 de dez.. 2016. Disponível em: http://www.planalto.gov.br/ccivil_03/_ato2015-2018/2016/lei/113409.htm. Acesso em: 21 set. 2010.

BRASIL. Decreto n ${ }^{\circ} 9.235$ de 15 de dezembro de 2017. Dispõe sobre o exercício das funções de regulação, supervisão e avaliação das instituições de educação superior e dos cursos superiores de graduação e de pós-graduação no sistema federal de ensino. Diário 
Oficial da União, Brasília, DF,18 de dez. de 2017. Disponível em http://www.planalto.gov.br/ccivil_03/_Ato2015-2018/2017/Decreto/D9235.htm.

Acesso em: 21 set. 2020.

BRASIL. Ministério da Educação. Instituto Nacional de Estudos e Pesquisas Educacionais Anísio Teixeira (Inep). Censo da Educação Superior 2018: Notas Estatísticas. Brasília, DF: Inep, 2019a. Disponível em: http://download.inep.gov.br/educacao_superior/censo_superior/documentos/2019/cens o_da_educacao_superior_2018-notas_estatisticas.pdf. Acesso em: 21 set. 2020.

BRASIL. Ministério da Educação. SIS/PROUNI: dados e estatísticas. 2019. Disponível em: http://prouniportal.mec.gov.br/dados-e-estatisticas. Acesso em: 21 dez. 2019.

CATANI, A. M.; OLIVEIRA, J. F. Educação superior no Brasil: reestruturação e metamorfose das universidades públicas. Petrópolis: Vozes, 2002.

COÊLHO, I. M. Universidade e ensino: treino ou formação. In: COÊLHO, I. M.; FURTADO, R. M. M. (Orgs). Universidade, cultura, saber e formação. Campina: Mercado de Letras, 2016.

DE KETELE, J.-M. Réformer l'éducation : travailler ensemble au bien commun en développant une intelligence collective. Revue internationale d'éducation de Sèvres, v. 83, p. 205$233,2020$.

DIAS SOBRINHO, J. Avaliação e transformações da educação superior brasileira (19952009): do provão ao Sinaes. Avaliação: Revista da Avaliação da Educação Superior, Campinas, Sorocaba, v. 15, n. 1, 2010.

DOURADO, L. F. Reforma do estado e as políticas para a educação superior no Brasil nos anos 90. Educação e Sociedade, Campinas, v. 23, n. 80, p. 234-252, 2002.

GARCON, F. Formation : l'autre miracle suisse. Lausanne : Presses polytcheniques et universitaires romandes, 2014.

GOHIER, C. Néo-libéralisme et éducation : quel(s) savoir(s) pour quel(s) pouvoir(s) ? Une question de valeurs ? In: M. BEHRENS ; A. BOURGOZ FROIDEVAUX (Eds). Réflexions sur l'avenir de l'éducation. Quelques jalons et perspectives (p. 107-122). Neuchâtel: IRDP Éditeur, 2016.

GRUENING, G. Origin and Theoritical Basis of New Public Management. International Public Management Journal, v. 4, n. 1-25, 2001.

HOOD, C. A Public Management for All Seasons. Public Administration, v. 69, n. 1, p. 3-19, $1991 / 2019$.

LERESCHE, J.-P. ; LAREDO, P.; WEBER, K. Recherche et enseignement supérieur face à l'internationalisation. France, Suisse et Union européenne. Presses polytechniques et universitaires romandes : Lausanne, 2009.

SGUISSARDI, V. Educação superior no Brasil - Refém do mercado ou quando a face privadomercantil se sobrepões à face pública do Estado. In: CHAVES, V. L. J.; MAUÉS, O. C.; HAGE, S. M. Expansão privado-mercantil na educação superior no Brasil. Campinas: Mercado de Letras, 2016. p. 17-56. 
SOUSA, J. V.; ROCHA, A. P. M. O. Repercussões da avaliação como instrumento de regulação da política educacional. In: RHOTEN, J. C.; SANTANA, A. C. M. (Orgs). Avaliação da educação: referências para uma primeira conversa. São Carlos: EdUFSCar, 2018. p. $157-172$.

SUISSE. LA POLITIQUE DE L'EDUCATION TERTIAIRE SUISSE. Rapport national de la Suisse. Accès en : https://www.hetsl.ch/uploads/media/rapport_federal_2002_01.pdf. Consulté le 31 août 2020.

SUISSE. SER [Secrétariat d'Etat à l'éducation et à la recherche]. Panorama de l'enseignement supérieur en Suisse. Bern, 2006.

SUISSE. AGENCE SUISSE D'ACCREDITATION ET D'ASSURANCE QUALITE. Accréditation institutionnelle. Guide/1.07.2015 (état au 1er août 2018). https://www.unige.ch/qualite/accreditation-universitaire/acrreditation-2022/. Consulté le 31 août 2020.

SUISSE. CSRE. RAPPORT SUR L'EDUCATION EN SUISSE. CRSE : Aarau, 2018.

SUISSE. SEFRI [Secrétariat d'Etat à la formation, à la recherche et à l'innovation]. La formation professionnelle en Suisse. Faits et chiffres 2019. Berne : SEFRI. 2019.

RECEBIDO: 29/06/2020

APROVADO: 03/10/2020

RECEIVED: 06/29/2020

APPROVED: $10 / 03 / 2020$

RECIBIDO: $29 / 06 / 2020$

APROBADO: 03/10/2020 